\title{
Correction to: Perceptions and sustainable actions under land degradation and climate change: the case of a remnant wetland in Mexico City
}

\author{
Laura Gómez Aíza ${ }^{1} \cdot$ Karina Rúlz Bedolla ${ }^{1}$ - Antonio M. Low-Pfeng ${ }^{2}$ (D) \\ Laura M. L. Vallejos Escalona ${ }^{3} \cdot$ Paola Massyel García-Meneses ${ }^{4}$ (D)
}

Published online: 26 June 2020

(c) Springer Nature B.V. 2020

\section{Correction to: Environment, Development and Sustainability https://doi.org/10.1007/s10668-020-00800-3}

Unfortunately, Karina Ruíz Bedolla name and affiliation have been published incorrectly in the original publication. They have been corrected.

Publisher's Note Springer Nature remains neutral with regard to jurisdictional claims in published maps and institutional affiliations.

The original article can be found online at https://doi.org/10.1007/s10668-020-00800-3.

Paola Massyel García-Meneses

paola.garcia@ecologia.unam.mx

Laura Gómez Aíza

galausa2001@yahoo.com.mx

Karina RuÍz Bedolla

karube@outlook.es

Antonio M. Low-Pfeng

alowxochi5@gmail.com

Laura M. L. Vallejos Escalona

lalis.vallejos@gmail.com

1 Consultor Independiente, Mexico City, Mexico

2 Universidad de Los Lagos, Centro i-mar, Camino a Chinquihue Km 6 Casilla 557, Puerto Montt, Región de Los Lagos, Chile

3 Subsecretaría de Programas de Alcaldías y Reordenamiento de la Vía Pública, Secretaría de Gobierno de la Ciudad de México, Fernando de Alva Ixtlixochitl \#185, Col Centro, Alcaldía Cuauhtémoc, Mexico City, Mexico

4 Laboratorio Nacional de Ciencias de la Sostenibilidad (LANCIS), Instituto de Ecología, Universidad Nacional Autónoma de México, Ciudad Universitaria Circuito exterior s/n anexo al Jardín Botánico Exterior, 06320 Mexico City, Mexico 